\title{
Characterization of liver specific promoters in a foamy viral vector pMD09
}

\author{
A. K. SINGH ${ }^{1}$, C. WEBER 2 , A. VARSHNEY', S. GUPTA ${ }^{1}$, S. N. KAZIM³ \\ M. G. SANAL ${ }^{1}$, A. RETHWILM ${ }^{2}$, S. K. SARIN ${ }^{4 *}$
}

\begin{abstract}
${ }^{1}$ Department of Molecular and Cellular Medicine, Institute of Liver and Biliary Sciences, New Delhi, India; ${ }^{2}$ Institut für Virologie und Immunbiologie, Universität Würzburg, Versbacher Str. 7, 97078 Würzburg, Germany; ${ }^{3}$ Hepatitis Research Lab, Centre for Interdisciplinary Research in Basic Sciences, Jamia Millia Islamia, New Delhi, India; ${ }^{4}$ Department of Hepatology, Institute of Liver and Biliary Sciences, New Delhi, India
\end{abstract}

Received May 21, 2018; revised September 6, 2018; accepted March 28, 2019

\begin{abstract}
Summary. - Foamy viruses (FVs) or spumaviruses are retroviruses that are explored as vectors for gene therapy. The good feature of foamy viruses is its broad tropism; however, their infections result in non-targeted gene expression. Here, we attempted to design the liver targeted viral gene delivery by employing liver specific gene promoters like albumin (ALB), transthyretin (TTR) and hepatitis B virus (HBV) promoters. We compared the relative gene expression of liver specific promoters versus the U3 promoter in liver cell line (HepG2) and non-liver cell lines: human fibrosarcoma cell line (HT1080), baby hamster kidney cell line (BHK), human embryonic kidney cell line (HEK 293T) and cervical cancer cell line (HeLa). We have found that the promoter exchange didn't affect viral assembly. The ability to drive gene expression was best with TTR promoter which was followed by HBV and ALB promoter. The use of TTR, HBV and ALB promoters are helpful in achieving liver specific gene expression.
\end{abstract}

Keywords: foamy virus; gene therapy; liver; albumin; transthyretin promoter; HBV promoter

\section{Introduction}

Gene therapy is still mostly an experimental technique. Several gene-therapy trials have been performed in the past two decades for inherited diseases, cancer and chronic infections, but only a few reported clear clinical benefits. On the other side, in some cases, individuals experienced severe adverse events related to the vectors. Gene transfer must overcome complex cellular and tissue barriers to deliver new genetic information into the target cell to drive proficient expression of a therapeutic molecule without disrupting essential regulatory mechanisms (Naldini, 2015). Despite all the obstacles, some recent clinical trials of gene therapy have shown remarkable therapeutic benefits and safety. Improved vector designs that enable the safe delivery of therapeutic

*Corresponding author. E-mail: sksarin@ilbs.in, shivsarin@gmail. com; phone: +011-4630-0000, mobile: +91-9873173140.

Abbreviations: $\mathrm{ALB}=$ albumin; $\mathrm{FV}(\mathrm{s})$ = foamy virus(es); $\mathrm{HBV}=$ hepatitis $\mathrm{B}$ virus; TTR $=$ transthyretin genes to specific cells, technologies for editing genes and correcting inherited mutations and the engagement of stem cells to regenerate tissues has already been studied. However, there is a still need to revisit the investigation of gene therapy.

Adeno associated virus based vectors are in the forefront of successful gene therapy clinical trials (Nathwani et al., 2011) However, major issues involved with adeno associated virus vector are immune mediated responses, limited cargo capacity and transient gene expression (Mingozzi and High, 2013).

In case of integrating viral vectors, such as lentiviruses, antibodies against the transgene and cell mediated immunity against the cells expressing the transgene compromise the success of gene therapy. Integrating virus such as retrovirus and lentivirus can be genotoxic because of its propensity to integrate to actively transcribed genes and their promoters (Rethwilm, 2007). Therefore, there is a need to develop new vectors and strategies for safer and effective gene therapy.

Foamy virus (FV) infects a wide range of mammals, including primates, felids, equines, ruminants and bats. En- 
dogenous FV sequences in sloths, the Cape golden mole, prosimians and the fossil fish coelacanth, indicate an FV-host relationship for over 400 million years (Trobridge, 2009). It may be noted that anti-FV antibodies are less prevalent in humans, since FVs or spuma-retroviruses are not usual in humans, although they are prevalent in nonhuman primates and in other mammals. They show efficient horizontal transmission in their host species but without pathological changes. FV vectors have several unique properties that make them a better choice for therapeutic gene transfer including a desirable safety profile, a broad tropism, a large transgene capacity, the ability to persist in quiescent cells and efficient and stable gene transfer (Erlwein and McClure, 2010, 2011).

Analysis of FV vector integration sites in vitro and in hematopoietic repopulating cells shows that they have a unique integration profile, proposing their better safety than in gamma-retroviruses or lentiviral vectors (Naldini, 2015). In a recent study comparing lentivirus and foamy virus, foamy vector proviruses were observed less often near to a gene or proto-oncogene transcription start sites compared to lentiviral vectors. The foamy vectors were more polyclonal with fewer dominant clones than the lentiviral vector group, and only lentiviral vectors integrated near known protooncogenes in dominant clones. Foamy retroviral vectors have a promising integration profile and are less prone to readthrough transcription than gamma retroviral or lentiviral vectors (Everson et al., 2016; Rethwilm, 2007) .

Foamy viruses can infect a wide range of tissues and therefore lack liver specificity. Liver gene therapy has implications in inherited liver diseases such as familial intra hepatic cholestasis, a1 anti-trypsin deficiency, Wilsons' disease, glycogen storage diseases, lipid storage diseases, Crigler Najjar syndrome, urea cycle disorders, familial dyslipidaemias, kidney diseases such as primary hyperoxalurea and bleeding disorders such as haemophilia (Geng et al., 2014). We have therefore chosen to test the promoters of two liver specific genes, albumin and transthyretin, as well as the promotor of hepatotropic HBV.

\section{Materials and Methods}

Vector constructs. The pMD09 vector is derived from the prototype (formerly human) FV and contains eGFP reporter gene under the control of retroviral U3 promoter. Three different vectors namely pMD09TTR, pMD09ALB and pMD09HBVCORE were constructed using standard recombinant DNA techniques by replacing the U3 promoter with Eco47III and BamHI restriction enzymes (Fig1). Promoters ALB and TTR were amplified from the genomic DNA isolated from HEK 293T cell lines and HBVCORE promoter was amplified from the plasmid pCF80 which contains tetramer of HBV genome using the specific sets of primers (Table1). In the first round of PCR for albumin promoter the
Table 1. List of oligonucleotide primers

\begin{tabular}{ll}
\hline Name & Primer \\
\hline ALB promoter F & 5'-TATGCCTGGTGAAGGTCAAG-3' \\
ALB promoter R & 5'-GGTTACCCACTTCATTGTGC- 3' \\
ALB F Eco47III & 5-GCTAGCGCTGTTTGCATCTGAGGCAA \\
& CATGAG-3' \\
ALB R BamHI & 5'-GCTAGCGCTGTTTGCATCTGAGGCAA \\
TTR F out & CATGAG-3' \\
TTR R out & 5'-GTCCTCTCCTGCACATTCTC-3' \\
TTRfw Eco47III & 5'-GCTAGCTCTCCTCTACCAAG-3' \\
& CAAG-3' \\
TTR rv out BamHI & 5'-CGGATCCGAGTGGGACCCATGGAACCAT \\
& GCTG-3' \\
HBV cpfw Eco47III & 5'-GCTAGCGCTGAACGCCCACCACTTCTT \\
& GC-3' \\
HBV cprvBamHI & 5'-GCGGATCCTGGTGCGCAAGACCAATT \\
& TATGC-3' \\
\hline
\end{tabular}

specific primer set used were 5'-TATGCCTGGTGAAGGTCAAG-3 forward primer and 5'-GGTTACCCACTTCATTGTGC-3' reverse primer. The product of first round of PCR was used as substrate for second round of PCR using the primer set 5-GCTAGCGCT GTTTGCATCTGAGGCAACATGAG-3' forward primer and 5'-GCTAGCGCTGTTTGCATCTGAGGCAACATGAG-3'reverse primer, which finally amplified the albumin promoter of $327 \mathrm{bp}$. The primer set used for the first round of PCR amplification for TTR promoter was 5'-GTCCTCTCCTGCACATTCTC-3' forward primer and 5'-GTGACCTCTCCTCTACCAAG-3' reverse primer. The first round of PCR product was further amplified by using 5'-GCTAGCGCTTGTTGACCCATGGAACCATCAAG-3' forward primer and 5'-CGGATCCGAGTGGACTTCTGTGATGGCTG-3' reverse primer which finally amplified the TTR promoter of $529 \mathrm{bp}$. HBV core promoter was amplified from plasmid pCF80 containing four copies of HBV genome in a single round of PCR using the primer set 5'-GCTAGCGCTGAACGCCCACCACTTCTTGC-3' forward primer and 5'-GCGGATCCTGGTGCGCAAGAC CAATTTATGC-3' reverse primer, which amplified the core promoter of $232 \mathrm{bp}$. After the amplification the liver specific promoter was ligated into pMD09 vector (without U3 promoter) using the T4 DNA ligase. Further, the clones were confirmed by restriction digestion of different vectors with specific restriction enzymes AflII, EcoNI, Psp14061.

Cell lines. We used five different cell lines HepG2 (human liver carcinoma cell line), Huh7 (human liver carcinoma cell line), HT1080 (human fibrosarcoma cell line), BHK (baby hamster kidney cell line), HEK 293T (human embryonic kidney cell line), and HeLa (cervical cancer cell line). HEK 293T cell lines were used to produce foamy viral vectors, BHK cell lines were used as transduction control (best for transduction by foamy viruses), HepG2 and Huh7 were used as liver specific cell lines and HT1080, HeLa were used as positive controls for human cancer cell lines. All the cell 
lines were provided by Institut fürVirologie und Immunbiologie, Universität Würzburg, Germany.

Cell culture experiments. HepG2 and Huh7 cell lines were maintained in DMEM (Life Technologies, Germany) containing 10\% fetal bovine serum (Life Technologies) and 1\% penicillin/streptomycin (Life Technologies). All other cell lines were maintained in MEM (Life Technologies) containing 10\% fetal bovine serum and $1 \%$ penicillin/streptomycin. For transfection experiments, $1 \times 10^{5}$ cells were plated in 24-well plates and co-transfected in triplicates with equimolar amounts of either pMD09TTR, pMD09ALB and pMD09CORE and pMD09 by using PIE (Polyethylenimine linear MW 25,000) (Polysciences Europe $\mathrm{GmbH}$, Germany) at $80 \%$ of the cell confluency. After $48 \mathrm{~h}$ of transfection, fluorescence intensity was measured using flouorimeter FLUOstar Omega (BMG Labtech, Germany).

Foamy viral vector production ( $P M D 09$ with U3 promoter and pMD09TTR, pMD09ALB and pMD09HBVCORE). Into a $20 \mathrm{~cm}$ culture plate $14.5 \times 10^{6}$ of HEK293T cells were seeded with $15 \mathrm{ml}$ of media. The following day confluent cells were transfected with four packaging plasmids, with the ratio of 10:5:1:1 (pMD09: pCZi-gag2: pCZi-pol: pCZHF-env) (Supplementary Fig. S1). In another set of experiments, plasmids pMD09TTR, pMD09ALB and pMD09HBVCORE were used for the transfection of cells containing the packaging constructs instead of pMD09. On the $5^{\text {th }}$ day, the transfection efficiency was determined under the fluorescence microscope (Fluorescence Microscope DMIRE S ${ }^{2}$, Germany). Supernatants were collected and filtered through $45 \mu \mathrm{m}$ filter and stored in the deep freezer. Different virus dilutions (1:10, 1:100, and 1:1000) were prepared and stored. $3 \times 10^{4}$ cells (BHK, HT1080, HepG2, and HeLa) were seeded in to 12 -well plates for the transduction with different foamy viral vectors produced by above mentioned experiment. On the second day transduction was performed with different dilutions of vectors and incubated at $37^{\circ} \mathrm{C}$. After $72 \mathrm{~h}$, the proportions (\%) of fluorescent cells were calculated using Flow jo software inflow cytometry (Becton Dickinson FACScan, BD Biosciences, Germany).

Flow cyotometry analysis. After $48 \mathrm{~h}$ of transfection media was removed from all the cells. The cells were washed with PBS and detached with trypsin for ten minutes. Media was added and cells were centrifuged at $1200 \mathrm{rpm}$ and resuspended in $100 \mu \mathrm{l}$ of $2 \%$ formaldehyde. The percentage of GFP positive cells were analyzed on a Becton Dickinson FACScan (BD Biosciences, Germany) using the Flow jo software against FL1-H.

\section{Results}

\section{Construction of liver specific FV constructs}

To generate the construct of foamy virus vectors with liver specific promoter, we have used three different liver specific promoters: transthyretin (TTR), albumin (ALB) and hepatitis B virus basal core promoter (HBV CORE) (Fig. 1). Liver specific promoter was exchanged with U3 promoter of foamy

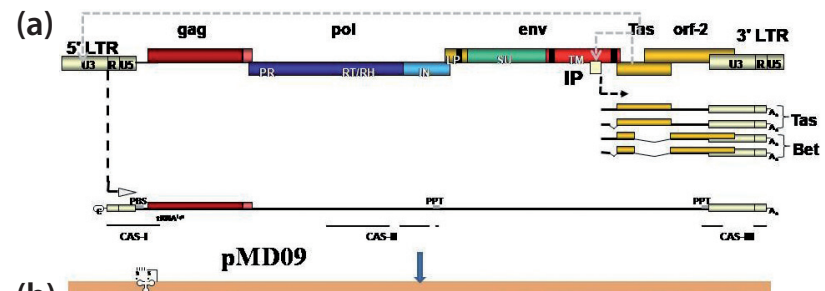

(b)

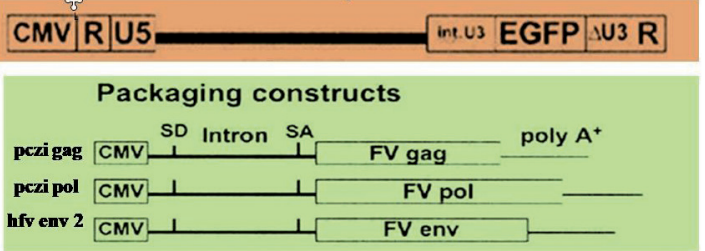

Fig. 1

Foamy virus and constructs used for transduction experiments. Representation of the arrangement of genes and transcripts of prototype foamy virus (PFV) (a) FV genome. Long terminal repeat (LTR), internal promoter (IP), cis-acting sequences (CAS), pol encapsidation signal (PES). (b) Plasmid and packaging constructs. pMD09 plasmid with CMV promoter and eGFP. Packaging constructs pCZi-gag2 containing FV gag gene for viral capsid, pCZi-pol containing FV pol gene for polymerase, pCZHFV-env containing $\mathrm{FV}$ env gene for envelope protein.

viral vector pMD09 using the standard cloning techniques (Fig. 2a,b). TTR and ALB promoters were amplified from genomic DNA isolated from HEK 293T cell using nested PCR approach. The albumin and transthyretin promoters were amplified in two rounds by polymerase chain reaction using specific primer sets. The product of first round of PCR was used as substrate for second round of PCR which finally amplified the albumin promoter of 327 bp (Fig. 2c) and TTR promoter of $529 \mathrm{bp}$ (Fig. 2e). HBV core promoter was amplified from plasmid pCF80 containing HBV genome in a single round of PCR yielding the core promoter of $232 \mathrm{bp}$ (Fig. 2d). After the amplification the liver specific promoter was ligated into pMD09 vector (without U3 promoter) using the T4 DNA ligase. The clones were confirmed by restriction digestion of different vectors with specific restriction enzymes (Fig. 2f).

\section{Transduction efficiency of foamy virus constructs} in different cell lines

The transduction efficiency of foamy virus was similar in all cell lines (BHK, HT1080, HepG2, and HeLa) tested, however the differences were not statistically significant (Fig. 3).

\section{Efficiency of liver specific promoters in different cell lines}

The promoter of the liver specific genes TTR, ALB and $\mathrm{HBV}$ versus $\mathrm{U} 3$ promoter were tested in cell lines (BHK, HEK, HT1080, HepG2 and HeLa) by measuring the expression of GFP which was expressed under the control of the 
(a)

\begin{tabular}{|c|c|c|c|c|c|}
\hline & $\begin{array}{l}\text { CAS I } \\
1.645\end{array}$ & $\underset{3869-5884}{\text { CAS II }}$ & \multicolumn{2}{|c|}{$\begin{array}{l}\text { SFFV U3 Promotor } \\
\text { mit Markergen }\end{array}$} & LTR \\
\hline CMV & $\Delta \mathrm{gag}$ & $\Delta$ pol & U3 & EGFP & $\Delta \mathrm{UB}$ \\
\hline \multirow[t]{2}{*}{ CMV } & $\Delta \operatorname{gag}$ & $\Delta$ pol & TTR & EGFP & $\Delta \mathrm{U3}$ \\
\hline & CAs 1 & $\begin{array}{c}\text { CAS II } \\
3869-5884\end{array}$ & & & LTR \\
\hline \multirow[t]{2}{*}{ CMV } & $\Delta \mathrm{gag}$ & $\Delta$ pol & ALB & EGFP & $\Delta \mathbf{U}_{3}$ \\
\hline & $\underset{1-645}{\operatorname{CAs} 1}$ & $\begin{array}{c}\text { CAs II } \\
3869-5884\end{array}$ & & & LTR \\
\hline CMV & $\Delta$ gag & $\Delta$ pol & CORE & EGFP & $\Delta \mathrm{U} 3$ \\
\hline
\end{tabular}

(c)

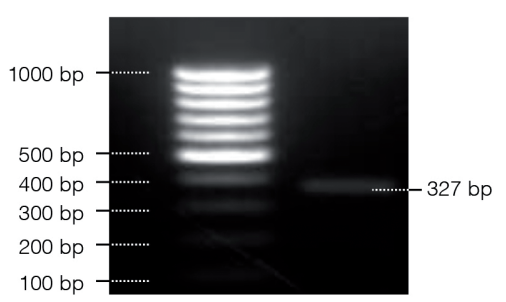

(b)

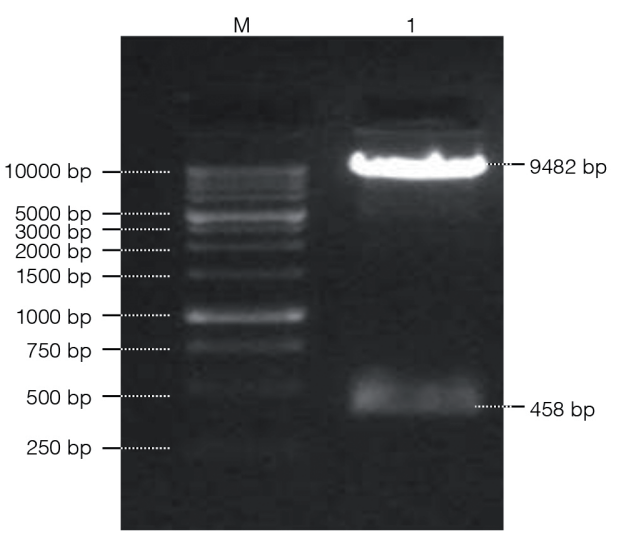

(e)

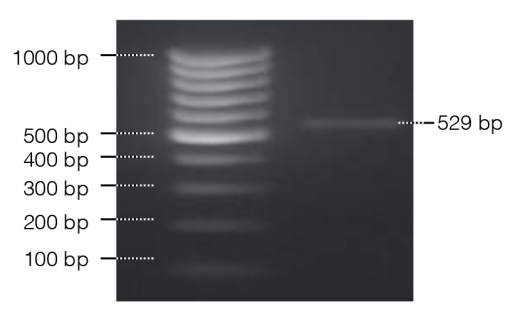

(f)

(d)

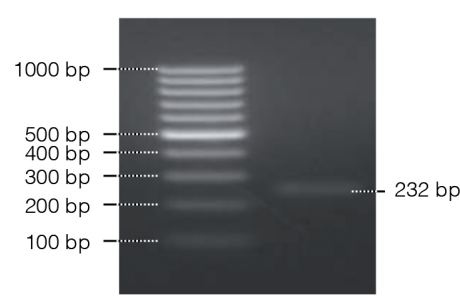

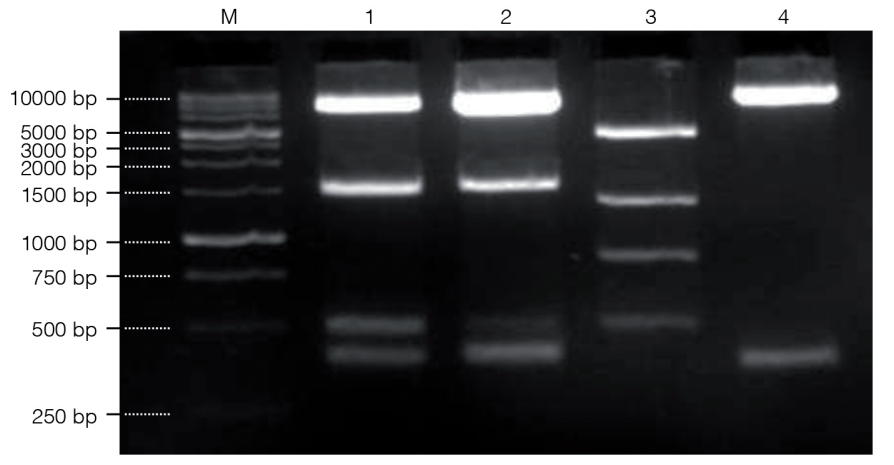

Fig. 2

Construction of liver specific foamy virus constructs

(a) Schematic diagram of foamy virus vectors and constructs. (b) Restriction digestion of pMD09 vector to replace U3 promoter. M = DNA marker from $250 \mathrm{bp}$ to $10000 \mathrm{bp}$, lane - pMD09 vector digested with BamHI and Eco47III restriction enzyme to yield U3 promoter (458 bp). (c) Amplification of ALB promoter from genomic DNA. M = DNA marker from $100 \mathrm{bp}$ to $1000 \mathrm{bp}$; lane - amplified ALB promoter (327 bp). (d) Amplification of HBV-CORE promoter from plasmid pCF80. M = DNA marker from $100 \mathrm{bp}$ to $1000 \mathrm{bp}$; lane - amplified core promoter (232bp). (e) Amplification of TTR promoter from genomic DNA. M = DNA marker from $100 \mathrm{bp}$ to $1000 \mathrm{bp}$; lane - amplified TTR promoter (529 bp). (f) Restriction digestion of different vectors to confirm the clones. $\mathrm{M}$ = DNA marker from $250 \mathrm{bp}$ to $10000 \mathrm{bp}$; lane 1 - pMD09U3 vector digested with AflII restriction enzyme, lane 2 - pMD09CORE vector digested with AflII restriction enzyme, lane 3 - pMD09TTR vector digested with EcoNI restriction enzyme, lane 4 - pMD09ALB vector digested with Psp14061 restriction enzyme.

afore mentioned promotors. As expected, the liver specific promoters resulted is a stronger expression of GFP in liver cell lines (HepG2 and HuH7) compared to BHK, HT1080, and HeLa (Fig. 4a,b). Among the liver specific promoters, TTR was relatively stronger than HBV and ALB. The promoter activity of TTR was about 8 times stronger when compared to $\mathrm{U} 3$ promoter in HepG 2 cells. The HBV and albumin promoter activities were, about 7 and 6 times stronger respectively, compared to U3 promoter activity in HepG2 cells. The expression of GFP was about $30 \%$ and $60 \%$ respectively, in 
Transduction efficiency

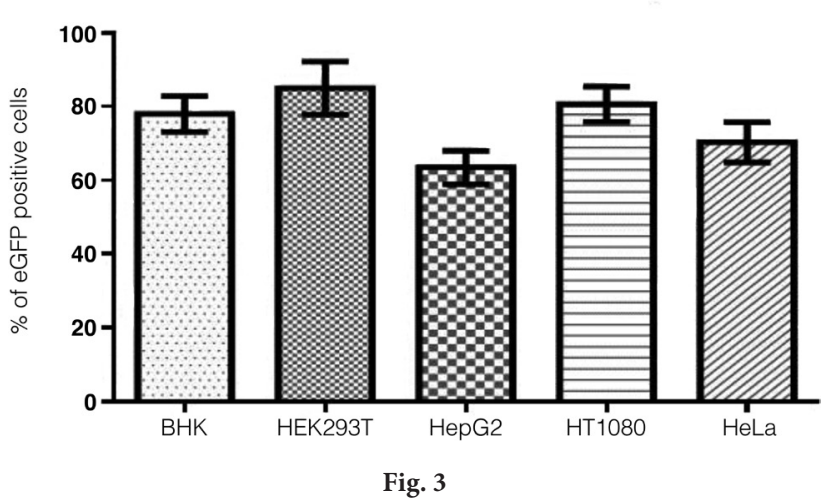

Transduction efficiency of foamy viral vector in different cell lines The transduction efficiency of foamy virus was similar in all cell lines (BHK, HT1080, HepG2, HEK293T and HeLa) tested. The differences were not statistically significant.

HeLa cells compared to the GFP expression in HepG2 cells under TTR and $\mathrm{U} 3$ promoters. The GFP expression was very weak in BHK, HEK and HT1080 cell lines under liver specific promoters compared to U3 promoter. However, the U3 promoter mediated expression was about 5 times stronger in BHK cell line than TTR promoter mediated GFP expression in liver cancer cells.

\section{Promotor exchange did not affect viral packaging}

The introduction of new promoters can affect the packaging efficiency of new vectors. To verify this, we produced the foamy viral vectors containing U3 (pMD09), TTR (pMD09TTR), HBV (pMD09HBVCORE), albumin (pMD09ALB) promoters in HEK 293T cells and transduced the BHK and HT1080 with these vectors and after $72 \mathrm{~h}$ of transduction, florescent cells were analyzed by flow cytometry. Results showed no significant difference between liver specific promoters with reference to the virus packaging efficiency (Fig. 5).

\section{Discussion}

Foamy virus vectors were shown to have an efficient gene delivery in variety of target cells (Lindemann and Rethwilm, 2011). In this study, we have characterized different promoters for liver specific gene therapy in foamy virus vector pMD09 (Kramer et al., 2003). In terms of the ability to drive gene expression it was found that TTR promoter was strongest followed by HBV promoter and albumin promoter. They were relatively liver (hepatocyte) specific because the relative gene expression driven by these promoters was 3 to 4 -fold more in hepatocyte cell line HepG2 compared to

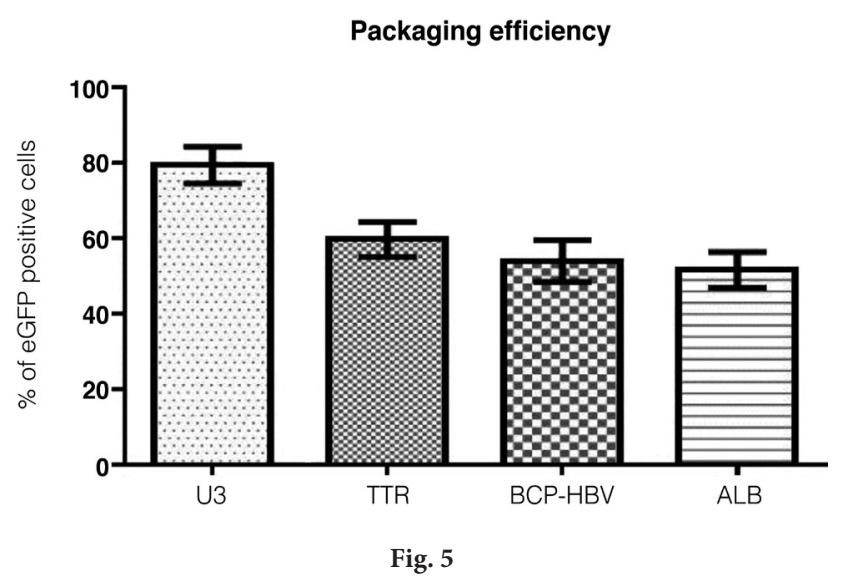

Packaging efficiency of liver specific promoters

There was no significant difference between liver specific promoters with reference to the virus packaging efficiency. Proportions (\%) of fluorescent cells were calculated by flow cytometry. pMD09U3 showed 79\% GFP positive cells whereas pMD09TTR, pMD09ALB and pMD09CORE constructs showed from $53 \%$ to $58 \%$ GFP positive cells.

BHK, HeLa, HEK and HT1080. It may be interesting to note that strong promoter U3 expression in HeLa cells is only 2 folds higher than that of TTR, HBV and ALB mediated gene expression. This may be explained by certain similarities which the cervical cancer cell line HeLa might have acquired over many decades of evolution. In fact, Chang cell line, which is used in Bio-Artificial Liver (BAL) reactors is derived from HeLa cells. The cell line Chang was originally thought to be derived from normal liver. The cell line has since been found to be indistinguishable from HeLa cells by STR PCR DNA profiling (Masters et al., 2001). Earlier reports also showed that liver targeted gene expression using liver-specific foamy virus promoters can infect a wide range of species and a wide range of tissues and found to be successful in gene therapy (Bauer et al., 2008; Erlwein and McClure, 2010; Trobridge, 2009; Weber et al., 2013). In future it may be possible to derive foamy virus which is organ specific through genetic engineering. However, currently, making use of liver specific promoters is the only way to make foamy viral gene therapy liver specific. Similarly, Geng et al. (2014) also showed liver specific gene therapy by using hepatocyte-specific $\alpha$-fetoprotein (AFP) enhancer/albumin promoter. We conclude that the use of TTR, HBV and ALB promoters can make foamy virus mediated gene expression relatively liver specific.

Acknowledgment. This work was supported by DST-DAAD project entitled Liver gene therapy by foamy virus vectors. Project No. INT/DAAD-159/2007.

Supplementary information is available in the online version of the paper. 
(a)
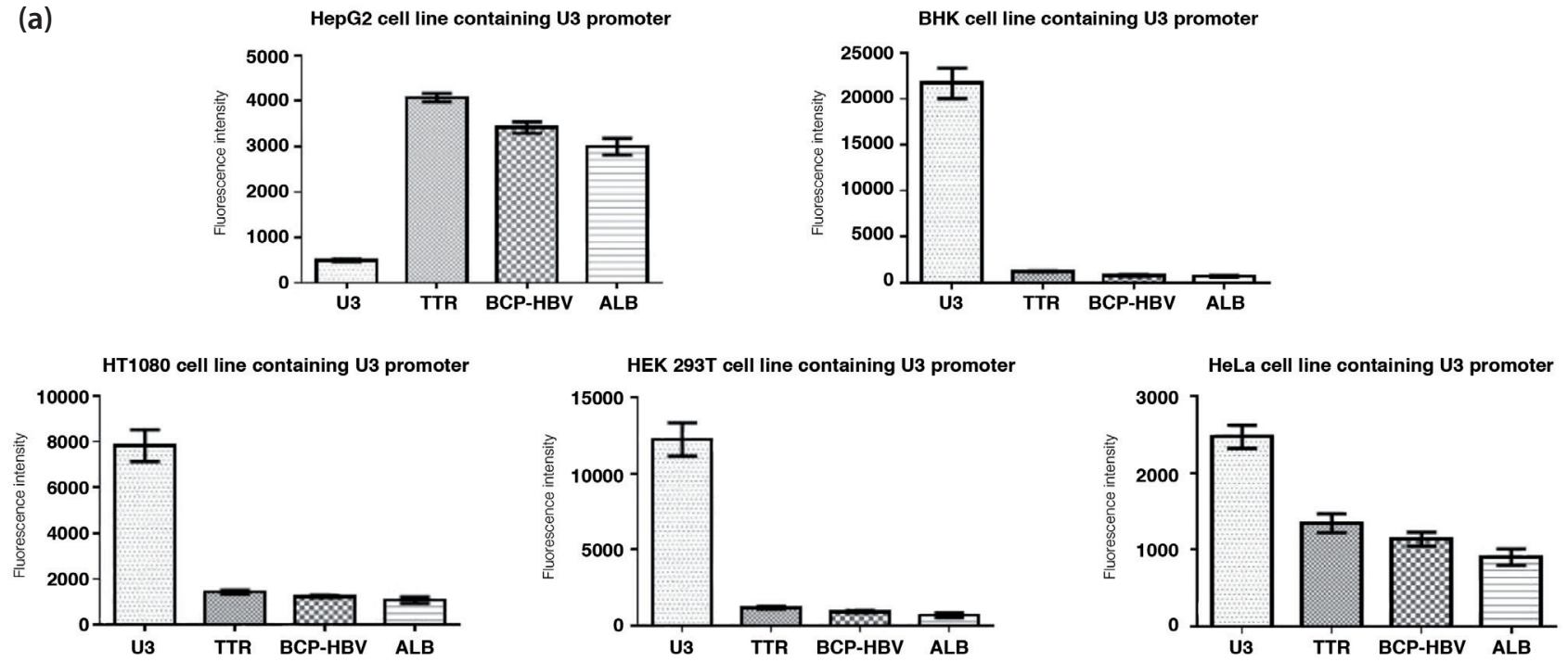

(b)
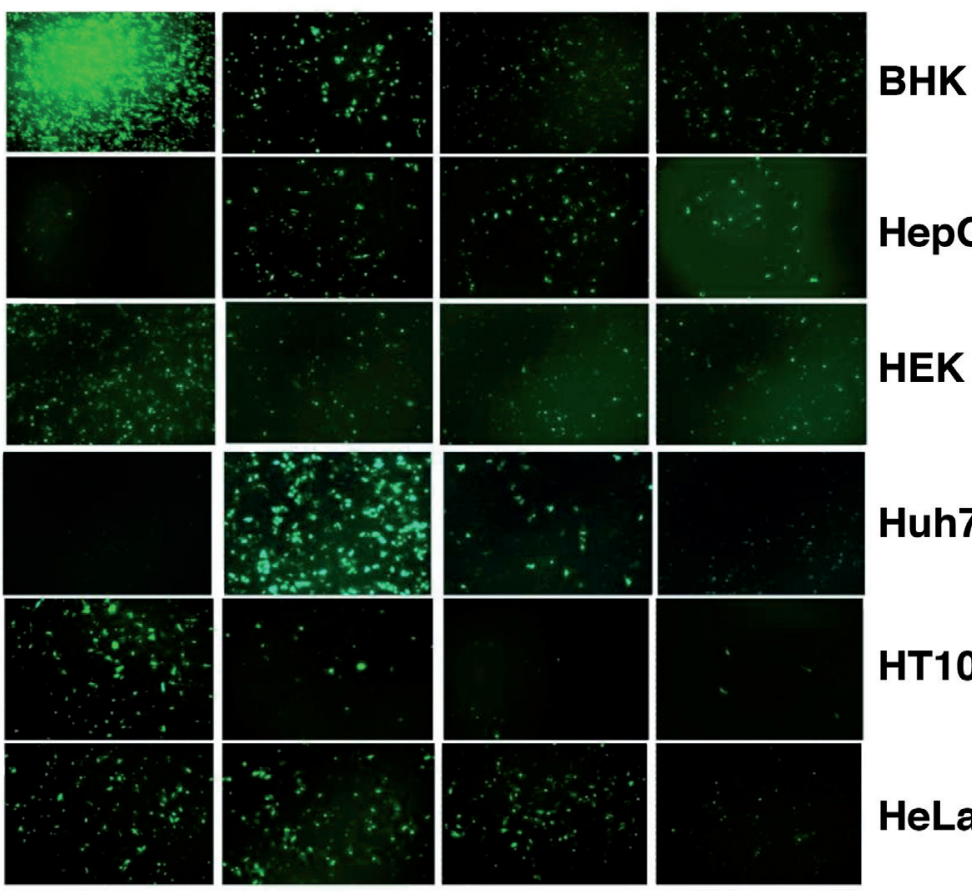

BHK

U3

TTR

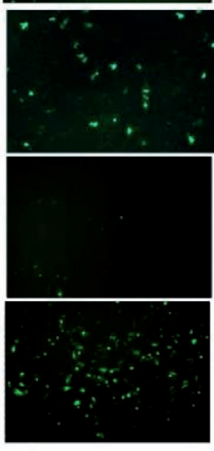

BCP-HBV

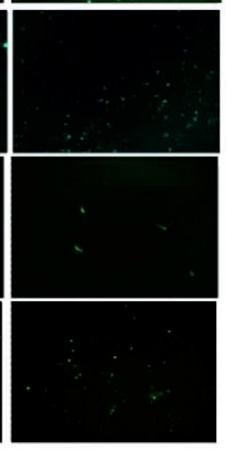

ALB

Fig. 4

Transfection efficiency of liver specific promoters

(a) The promoter of the liver specific genes transthyretin (TTR), albumin (ALB) and hepatitis B virus (HBV) versus U3 promoter expression were tested in cell lines (BHK, HEK 293T, HT1080, HepG2 and HeLa) using flow cytometry. (b) Liver specific promoters resulted in a higher transfection efficiency correlated with expression of GFP in HepG2 and Huh7 compared to BHK, HT1080, HEK 293T, and HeLa analysed using florescence microscope.

\section{References}

Bauer TR Jr., Allen JM, Hai M, Tuschong LM, Khan IF, Olson EM, Adler RL, BurkholderTH, Gu YC, Russell DW, Hickstein DD (2008): Successful treatment of canine leukocyte ad- hesion deficiency by foamy virus vectors. Nat. Med. 14, 93-97. https://doi.org/10.1038/nm1695

Erlwein O, McClure M (2011): Gene delivery the key to gene therapy: the case for foamy viruses. Ther. Deliv. 2, 681-684. https://doi.org/10.4155/tde.11.38 
Erlwein O, McClure MO (2010): Progress and prospects: foamy virus vectors enter a new age. Gene Ther. 17, 1423-1429. https://doi.org/10.1038/gt.2010.95

Everson EM, Olzsko ME, Leap DJ, Hocum JD, Trobridge GD (2016): A comparison of foamy and lentiviral vector genotoxicity in SCID-repopulating cells shows foamy vectors are less prone to clonal dominance. Mol. Ther. Methods Clin. Dev. 3, 16048. https://doi.org/10.1038/mtm.2016.48

Geng J, Wei H, Sun R, Tian Z (2014): Construction and application of a novel hepatocyte directed vector to simultaneous knockdown and overexpression of multiple genes. Liver Int. 34, e246-56. https://doi.org/10.1111/liv.12336

Kramer MG, Barajas M, Razquin N, Berraondo P, Rodrigo M, Wu C, Qian C, Fortes P, Prieto J (2003): In vitro and in vivo comparative study of chimeric liver-specific promoters. Mol. Ther. 7, 375-385. https://doi.org/10.1016/S15250016(02)00060-6

Lindemann D, Rethwilm A (2011): Foamy virus biology and its application for vector development. Viruses 3, 561-585. https://doi.org/10.3390/v3050561

Masters JR, Thomson JA, Daly-Burns B, Reid YA, Dirks WG, Packer P, Toji LH, Ohno T, Tanabe H,Arlett CF, Kelland LR, Harrison M, Virmani A, Ward TH, Ayres KL, Debenham PG (2001): Short tandem repeat profiling provides an international reference standard for human cell lines. Proc. Natl. Acad. Sci. USA 98, 8012-8017. https://doi. org/10.1073/pnas.121616198
Mingozzi F, High KA (2013): Immune responses to AAV vectors: overcoming barriers to successful gene therapy. Blood 122, 23-36. https://doi.org/10.1182/blood-2013-01-306647

Naldini L (2015): Gene therapy returns to centre stage. Nature. 526, 351-360. https://doi.org/10.1038/nature15818

Nathwani AC, Tuddenham EG, Rangarajan S, Rosales C, McIntosh J, Linch DC, Chowdary P, Riddell A, Pie AJ, Harrington C, O'Beirne J, Smith K, Pasi J, Glader B, Rustagi P, Ng CY, Kay MA, Zhou J, Spence Y, Morton CL, Allay J, Coleman J, Sleep S, Cunningham JM, Srivastava D, BasnerTschakarjan E, Mingozzi F, High KA, Gray JT, Reiss UM, Nienhuis AW, Davidoff AM (2011): Adenovirus-associated virus vector-mediated gene transfer in hemophilia B. N. Engl. J. Med. 365, 2357-2365. https://doi.org/10.1056/ NEJMoa1108046

Rethwilm A (2007): Foamy virus vectors: an awaited alternative to gammaretro- and lentiviral vectors. Curr. Gene Ther. 7, 261-271. https://doi.org/10.2174/156652307781369092

Trobridge GD (2009): Foamy virus vectors for gene transfer. Expert. Opin. Biol. Ther. 9, 1427-1436. https://doi. org/10.1517/14712590903246388

Weber C, Armbruster N, Scheller C, Kreppel F, Kochanek S, Rethwilm A, Steinert AF (2013): Foamy virus-adenovirus hybrid vectors for gene therapy of the arthritides. J. Gene Med. 15, 155-167. https://doi.org/10.1002/jgm.2705 


\title{
Supplementary information
}

\section{Characterization of liver specific promoters in a foamy viral vector pMD09}

\author{
A. K. SINGH ${ }^{1}$, C. WEBER ${ }^{2}$, A. VARSHNEY ${ }^{1}$, S. GUPTA ${ }^{1}$, S. N. KAZIM ${ }^{3}$, \\ M. G. SANAL ${ }^{1}$, A. RETHWILM ${ }^{2}$, S. K. SARIN ${ }^{4 *}$
}

${ }^{1}$ Department of Molecular and Cellular Medicine, Institute of Liver and Biliary Sciences, New Delhi, India; ${ }^{2}$ Institut für Virologie und Immunbiologie, Universität Würzburg, Versbacher Str.7, 97078 Würzburg, Germany; ${ }^{3}$ Hepatitis Research Lab, Centre for Interdisciplinary Research in Basic Sciences, Jamia Millia Islamia, New Delhi, India; ${ }^{4}$ Department of Hepatology, Institute of Liver and Biliary Sciences, New Delhi, India

Received May 21, 2018; revised September 6, 2018; accepted March 28, 2019

Fig. S1. Foamy virus vector constructs

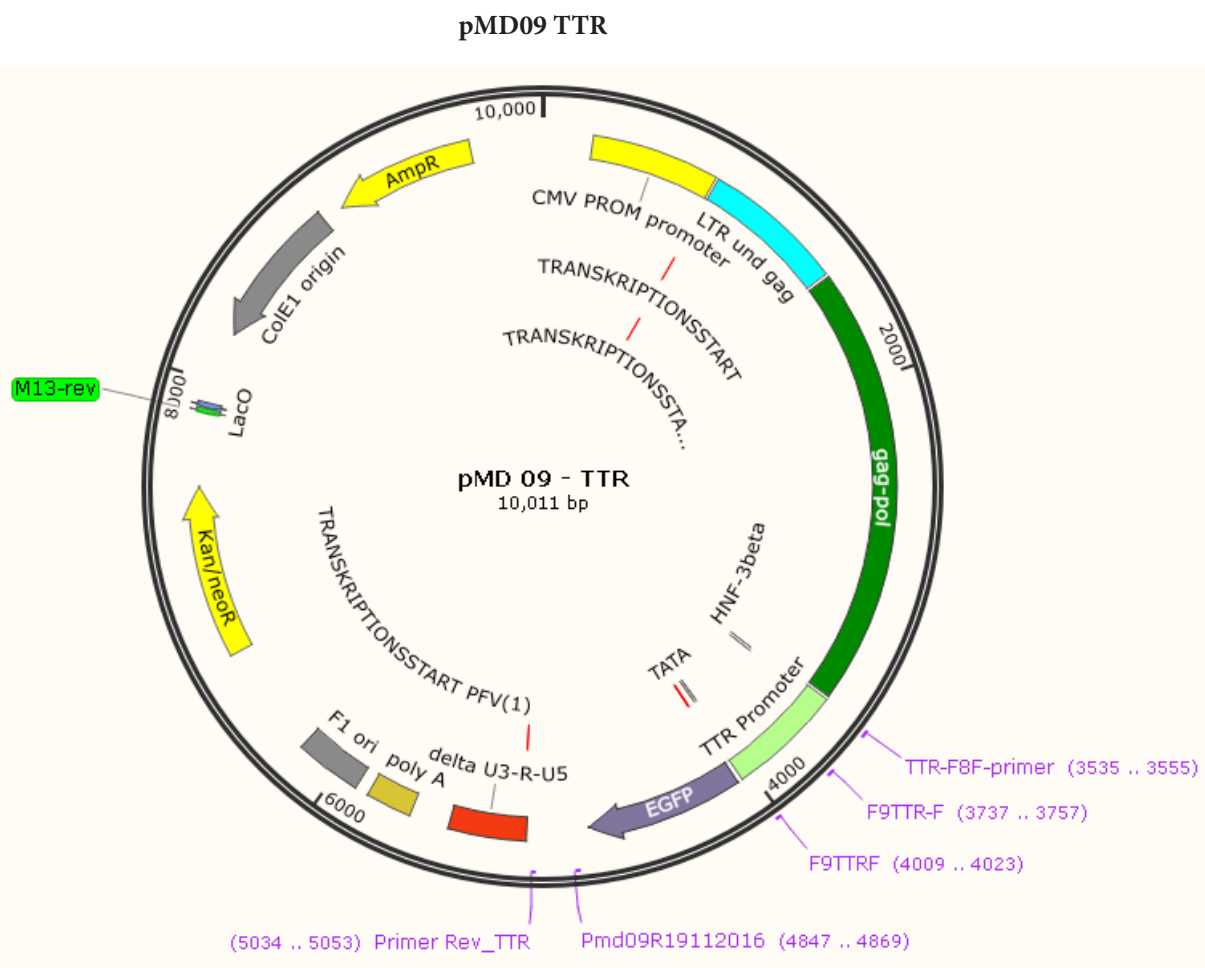

*Corresponding author. E-mail: sksarin@ilbs.in, shivsarin@gmail.com; phone: +011-4630-0000, mobile: +91-9873173140. 

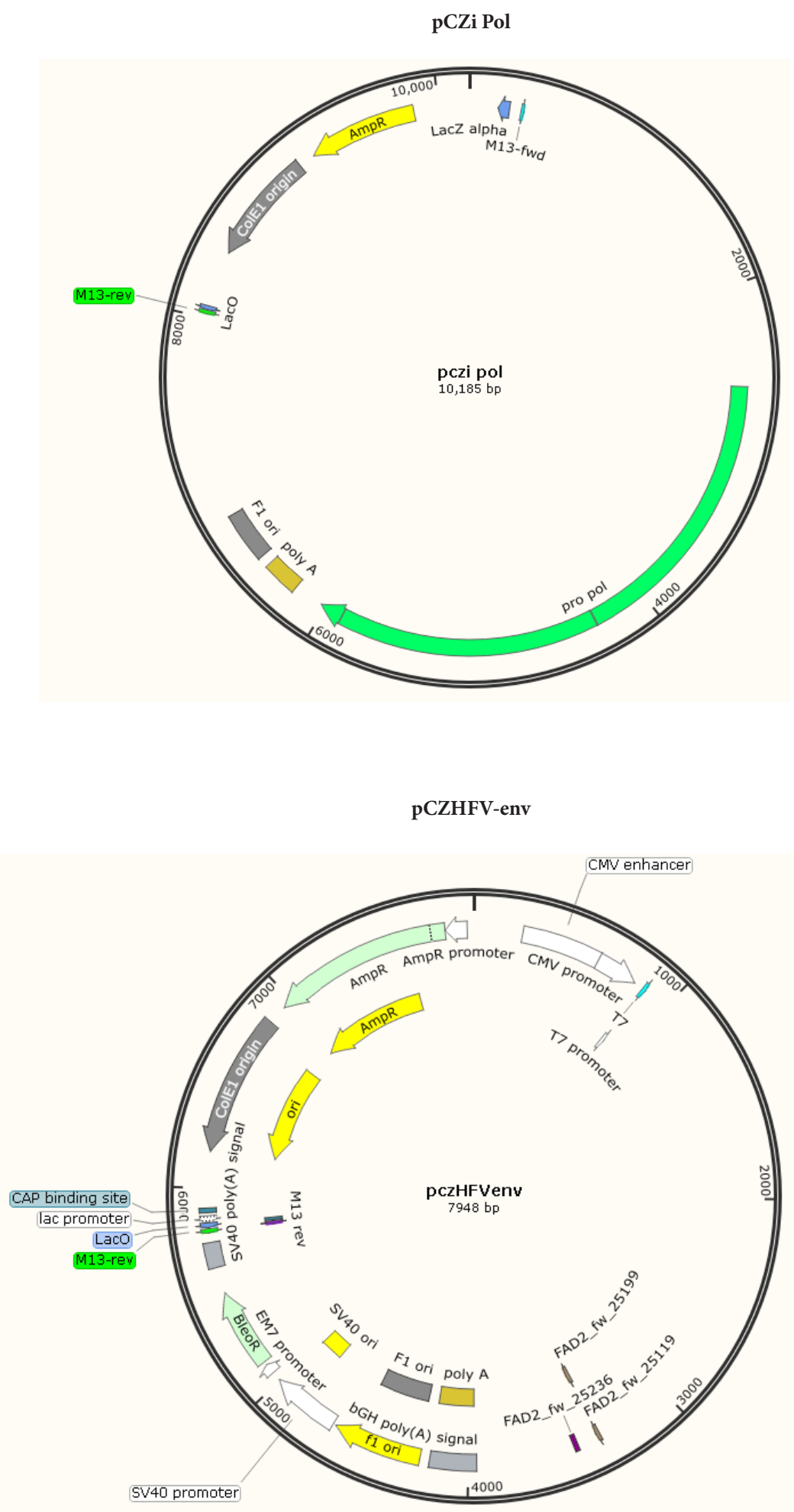


$$
8
$$

